OPEN ACCESS

Edited by:

Anna Kathleen Coussens,

University of Cape Town, South Africa

Reviewed by:

Subash Babu,

International Centers for Excellence in

Research (NIH), India Wendy Burgers,

University of Cape Town, South Africa

*Correspondence: Katharina Ronacher Katharina.Ronacher@mater.uq.edu.au

Specialty section: This article was submitted to Microbial Immunology, a section of the journal

Frontiers in Immunology

Received: 16 April 2018 Accepted: 06 September 2018 Published: 25 September 2018

Citation:

Ronacher K, Sinha R and Cestari M (2018) IL-22: An Underestimated

Player in Natural Resistance to

Tuberculosis? Front. Immunol. 9:2209

doi: 10.3389/fimmu.2018.02209

\section{IL-22: An Underestimated Player in Natural Resistance to Tuberculosis?}

\author{
Katharina Ronacher ${ }^{1,2 *}$, Roma Sinha ${ }^{2}$ and Michelle Cestari ${ }^{2}$ \\ ${ }^{1}$ Division of Molecular Biology and Human Genetics, Faculty of Medicine and Health Sciences, SAMRC Centre for \\ Tuberculosis Research, DST-NRF Centre of Excellence for Biomedical Tuberculosis Research, Stellenbosch University, Cape \\ Town, South Africa, ${ }^{2}$ Infection, Immunity and Metabolism Group, Translational Research Institute, Mater Research Institute \\ and The University of Queensland, Brisbane, QLD, Australia
}

Approximately $10 \%$ of individuals latently infected with Mycobacterium tuberculosis (Mtb) develop active tuberculosis (TB) during their lifetime. Although it is well recognized that T-helper 1 immune responses are crucial for containing latent TB infection, the full array of host factors conferring protective immunity from TB progression are not completely understood. IL-22 is produced by cells of the innate and adaptive immune system including innate lymphoid cells, and natural killer cells as well as T lymphocytes (Th1, Th17, and Th22) and binds to its cognate receptor, the IL-22R1, which is expressed on non-hematopoietic cells such as lung epithelial cells. However, recent studies suggest that Mtb induces expression of the IL-22R1 on infected macrophages and multiple studies have indicated a protective role of IL-22 in respiratory tract infections. Reduced concentrations of circulating IL-22 in active TB compared to latent TB and decreased percentages of Mtb-specific IL-22 producing T cells in TB patients compared to controls designate this cytokine as a key player in TB immunology. More recently, it has been shown that in type 2 diabetes (T2D) and TB co-morbidity serum IL-22 concentrations are further reduced compared to TB patients without co-morbidities. However, whether a causative link between low IL-22 and increased susceptibility to TB and disease severity of TB exists remains to be established. This review summarizes the contribution of IL-22, a potentially under-appreciated key player in natural resistance to TB, at the interface between the immune response to Mtb and the lung epithelium.

Keywords: tuberculosis, Mycobacterium tuberculosis, interleukin-22, IL-22R1, T lymphocytes, respiratory infections

\section{INTRODUCTION}

A quarter of the human population is infected with M. tuberculosis (Mtb) (1) of which $\sim 10 \%$ will develop the active and contagious form of tuberculosis (TB) during their lifetime (2). Various intrinsic and extrinsic factors determine the natural course of mycobacterial infection, and resistance vs. susceptibility to disease progression. These factors include host genetic susceptibility (3), virulence of the infecting strain (4) and presence of acquired immune deficiencies such as HIV infection and type 2 diabetes (T2D) (5). The role of IL-22 during the host defense against Mtb is poorly understood. The subsequent sections highlight our current knowledge of the protective function of IL-22 during respiratory tract infections, including TB. 


\section{SOURCE AND TARGETS OF IL-22}

IL-22 is produced by cells of the innate as well as the adaptive immune system including tissue resident innate lymphoid cells (ILCs), NK cells, macrophages, NKT cells, activated Th1, Th17, and Th22 cells as well as Tc-cell subsets and $\gamma \delta \mathrm{T}$ cells (6). Alveolar macrophages from both humans and mice are also able to produce and release IL-22 (7). In mice, antigen-specific IL-22 production is driven by Th1 and Th17 cells, but only a small subset of Th17 cells produce IL-22 in humans. In contrast to mice, humans have a distinct subset of T helper cells, called Th22 cells, which produce IL-22 and TNF $\alpha$. Unlike Th1 and Th17 subsets, human Th22 cells, which were initially characterized in skin neither produce IL-17 nor IFN $\gamma$ (8). Apart from secreting IL-22, Th22 cells can also express granzymes, IL-13 and increased levels of Tbet showing a remarkable plasticity to skew the immune response toward pro- or anti-inflammatory depending on the Th1 or Th2 stimulus in vitro (9).

IL-22 binds to its heterodimeric receptor complex consisting of the IL-22R1 and the IL-10R2 to activate the JAK-STAT signaling pathways (10). The IL-22R is present on epithelial cells of the lung, gut and skin, the liver, pancreas, and kidneys. It is not expressed on hematopoietic cells, neither in resting/naïve nor activated macrophages, $\mathrm{T}$ or $\mathrm{B}$ cells, nor the human monocyte THP-1 cell-line $(11,12)$. However, three independent studies reported that Mtb induces expression of the IL-22R1 in infected macrophages (13-15). The significance of this is discussed in the next section.

A $\mathrm{T}$ cell-derived soluble IL-22 binding protein (IL-22BP), which shares sequence homology with the extracellular domain of the membrane bound IL-22R1, acts as endogenous inhibitor of IL-22 by preventing its binding to the IL-22R1. Activation of the IL-22 signaling pathway in epithelial cells results in epithelial tissue proliferation, regeneration, and healing, therefore this cytokine plays an important role in protection from infectioninduced tissue damage at mucosal surfaces (10). IL-22 induces expression of the chemokines CXCL1 and CXCL5 in bronchial epithelia in a Klebsiella pneumoniae infection model (16), but reduces CXCL8, a neutrophil attracting chemokine, in A459 human lung carcinoma cells (17). Most importantly, IL-22 stimulates the production of antimicrobial peptides such as $\beta$ defensins, the S100 family of peptides, Reg $3 \beta$ and $\gamma$, lipocalin2 , calprotectin and calgranulin A in various cell types (18-21), thereby controlling bacterial growth and reducing the risk of secondary bacterial infections after viral injury (22).

\section{IL-22 AS IMMUNE-MODULATOR TO INHIBIT MYCOBACTERIAL GROWTH}

In addition to the well-described effect of IL-22 on epithelial cells the recent reports that Mtb induces expression of the IL$22 \mathrm{R} 1$ on macrophages, the primary host immune cells targeted by mycobacteria, is particularly intriguing. Treerat and colleagues report IL-22R1 positive macrophages by immunohistochemistry in granulomas of HN878 infected mice, but whether this positive signal is due to HN878 induced IL-22R1 expression on macrophages or through ingestion of IL-22R1 positive epithelial cell debris by the lung macrophages remains to be confirmed (15). Two previous studies report a modest induction of IL-22R1 expression on macrophages after stimulation with Mtb H37Rv and Erdman by flow cytometry $(13,14)$. Upregulation of the IL-22R1 in infected macrophages may be a host-mechanism to combat the infection, as there is growing evidence that IL-22 can modulate mycobacterial growth within macrophages.

In initial experiments Dhiman et al. observed that Mtbinfected human monocytes induce production of IL-22 by cocultured autologous NK cells in a IL-15 and IL-23 dependent manner. This NK mediated IL-22 production resulted in reduction of intra-macrophagic bacteria and was reversed through neutralization of IL-22 suggesting that the mycobacterial growth inhibition is at least in part attributable to IL-22 (13). In subsequent experiments by the same group exogenous addition of recombinant IL-22 (rIL-22) to infected macrophages promoted phagolysosomal fusion and reduced bacterial burden (23). The anti-mycobacterial activity of IL-22 was mediated through increased expression of the anti-microbial peptide calgranulin A and siRNA knock down of calgranulin A abrogated the IL-22 dependent mycobacterial containment in monocyte derived macrophages (23). An additional mechanism by which IL-22 may contribute to reduction in mycobacterial burden is the observed increased TNF $\alpha$ production by Mtb-infected bone marrow-derived macrophages when pre-treated with rIL-22 (15), however this mechanism requires confirmation through $\mathrm{TNF} \alpha$ neutralization experiments.

An unusual observation that a subset of $\mathrm{CD} 4^{+} \mathrm{T}$-cells in Mtbinfected humans and macaques retain IL-22 at the cell membrane instead of secreting it was reported by Zeng et al. who speculated that a membrane-bound IL-22 may enjoy longer half-life. The authors show that $\mathrm{IL}-22^{+} \mathrm{CD} 4^{+} \mathrm{T}$ cells reduce intra-macrophagic mycobacteria by direct cell-to-cell contact, however whether the anti-mycobacterial effect is indeed mediated by direct interaction of membrane-bound IL-22 on T cells with the IL-22R1 on macrophages remains to be corroborated with additional data (14).

The responsiveness of macrophages to IL-22 has also been shown in a different context, where IL-22 modulates cholesterol efflux from macrophages (24). This may have implications for control of mycobacteria, which catabolize host sterols to sustain a persistent infection (25). A summary of our current knowledge of the actions of IL-22 is shown in Figure 1.

\section{THE ROLE OF IL-22 IN EXPERIMENTAL ANIMAL MODELS OF LUNG INFECTION}

In an experimental murine model of Streptococcus pneumoniae rapid accumulation of IL-22 producing ILC3 in the lungs were observed and associated with protection from lethal infection (26). IL-22 $2^{(-/-)}$mice had greater streptococcal burden compared to wild-type mice and administration of rIL-22 reduced bacterial burden (27). Similarly, reduction of IL-22 production by depletion of ILCs in a Pseudomonas aeruginosa murine model induced lung injury was associated with reduced survival (28) 


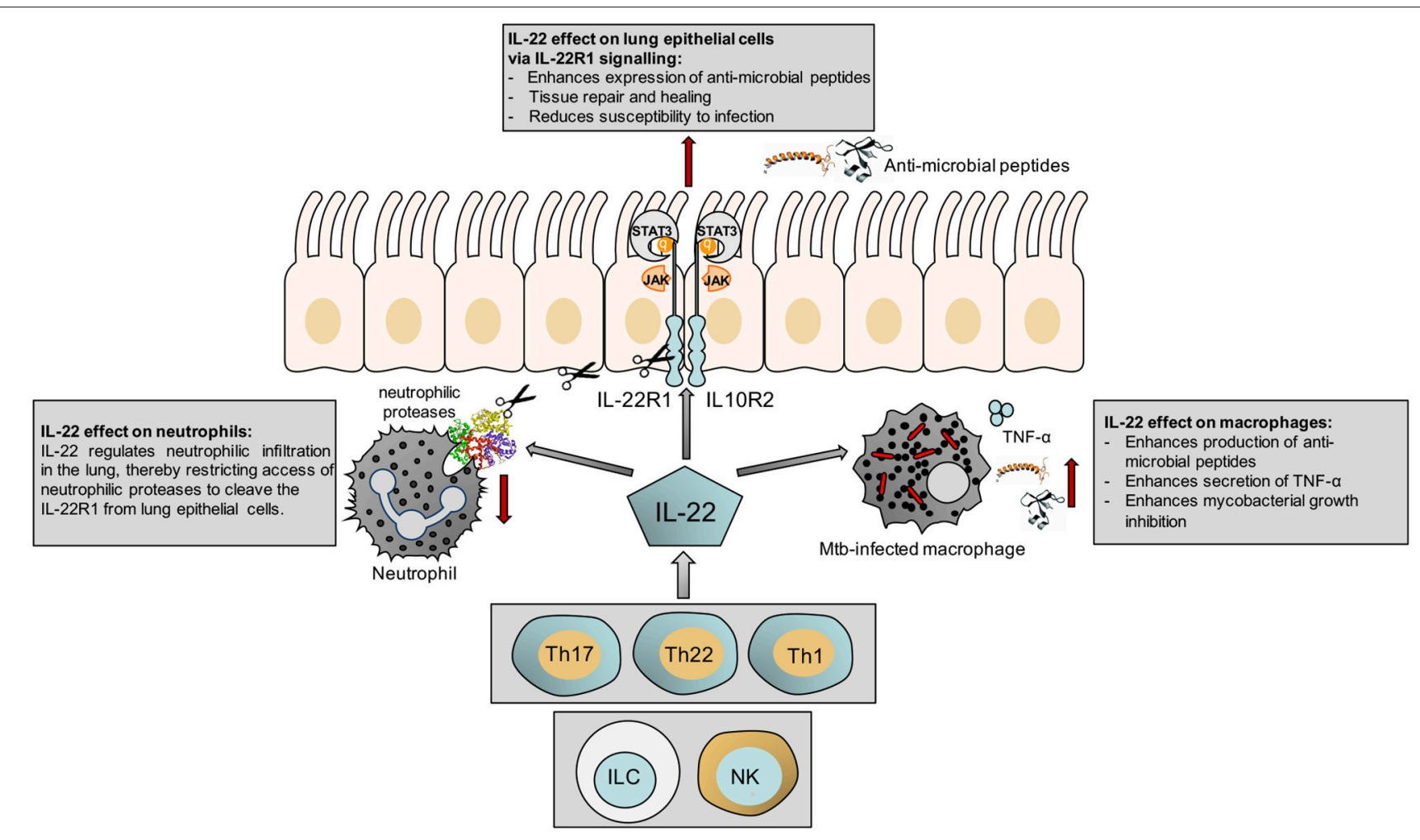

FIGURE 1 | Schematic illustrating the effect of IL-22 on the epithelium, macrophages and neutrophils.

pointing toward a host-protective role of IL-22 in both lung infection models. Interestingly, antibody-based neutralization of IL-22 led to increased neutrophilic infiltration and susceptibility to $P$. aeruginosa (29). This observation is consistent with the finding that IL-22 reduces expression of the neutrophil attracting chemokine CXCL8 from lung epithelial cells (17) and therefore a lack of IL-22 likely increases CXCL8, which in turn may drive the neutrophilic infiltration.

Neutrophilic proteases were previously shown to cleave the IL-22R1 on human bronchial epithelial cells and impair IL22 -dependent $\beta$-defensin expression, potentially contributing to pathogen replication (30). Administration of rIL-22 or neutralization of IL-22BP resulted in a decrease in lung damage and increased survival (29). Interestingly, $P$. aeruginosa has developed an immune-evasion strategy by secreting a serine protease which cleaves IL-22 resulting in its degradation, thereby weakening tissue repair and the anti-microbial defense (30). It will be interesting to investigate whether Mtb has acquired similar immune-evasion strategies and possesses proteases which cleave IL-22 and thus impair the IL-22R signaling pathway and host tissue repair. A murine model of Haemophilus influenzae infection further confirmed the beneficial effects of IL-22 observed in $P$. aeruginosa infected mice, where IL-22(-/-) mice had increased bacterial burden and administration of exogenous IL-22 boosted bacterial clearance and limited lung tissue damage (31). IL-22 secretion by Th17 cells is crucial for control of the Gram-negative pulmonary pathogen $K$. pneumoniae and promotion of lung epithelial cell proliferation (16). IL-22 also reduces lung inflammation during influenza $\mathrm{A}$ virus infection and protect against secondary bacterial infection (22). In summary, there is evidence from various lung infection models that IL-22 plays a protective effect on host mucosal surfaces, whereas the effect of IL-22 on bacterial clearance appears to be pathogen-specific.

Mycobacterial infection models using IL-22(-/-) mice have yielded conflicting results. IL-22 $2^{(-/)}$mice infected with both high and low dose H37Rv had comparable pro-inflammatory cytokine profiles in the lung as wild-type C57BL/6 mice with exception of increased IL-6 and reduced MMP-9 and CXCL10 (32). Recruitment of macrophages and granulocytes to the lung were similar between IL-22(-/-) and wild-type mice and there were no significant differences in bacterial burden and survival. Similar to the studies in IL-22(-/-) mice, administration of an IL-22 neutralizing antibody to wild-type mice 12 weeks post-infection did not compromise survival or alter bacterial burden (33). However, the timing of anti-IL22 administration may be crucial as the $P$. aeruginosa infection model suggests that elevated IL-22 concentrations prior to infection are important for conveying the protective effects (29). The Mtb infection studies in IL-22 $2^{(-/-)}$mice by Behrends and colleagues were carried out with $\mathrm{H} 37 \mathrm{Rv}$ and different results were obtained when the knock out mice were infected with HN878 (15). Mtb HN878 infection induced IL-22 production via a TLR2 and IL-1 $\beta$-dependent pathway and in this model IL-22 plays 
an important role in recruitment of myeloid cells to the lung (15). IL-22 $2^{(-/-)}$mice were more susceptible and exhibited higher bacterial burden during the chronic stage of HN878 infection 100 days post-infection, whereas no differences in susceptibility were observed during the acute phase 30 days postinfection. Therefore, these data suggest that susceptibility of IL$22^{(-/-)}$mice to mycobacteria is largely driven by the infection stage (acute vs. chronic) and the mycobacterial strain $(\mathrm{H} 37 \mathrm{Rv}$, Erdman vs. HN878). Strain specific differences in eliciting an IL-22 response have also been shown in PBMCs from TB patients stimulated with cell wall extracts from Mtb HN878, which resulted in greater production of IL-22 compared to $\mathrm{H} 37 \mathrm{Rv}$ cell wall extracts (15). In addition, as the timing of exogenous administration of IL-22 appears to be important from other infection models, further studies in IL-22(-/-) mice with administration of IL-22 prior to Mtb infection are required. Such studies are however complicated by the short half-life of IL-22 and its off-target effects on mucosal tissues other than the lung.

In a non-human primate model, Mtb infection resulted in reduced IL-22 mRNA expression in peripheral blood but increased expression in the lymphocytes of the lungs, bronchial lymph nodes, and the spleen (34). These observations from primates are consistent with human studies where elevated IL-22 protein was found at the site of disease in human broncho-alveolar lavage fluid (BALF) (35-37) as well as increased percentages of IL-22 ${ }^{+} \mathrm{CD} 4{ }^{+} \mathrm{T}$ cells in BALF compared to blood (38). Although elevation of IL-22 in primates was associated with severe TB, it is not clear whether IL-22 production is induced as a consequence of enhanced inflammation to counteract immunopathology or directly contributes to pathology itself. IL22 producing $\mathrm{T}$ cells were also observed in BALF from Mtbinfected compared to un-infected primates and were visualized in TB granulomas by immunohistochemistry (39). Furthermore, IL22 expression was also found in lung and lymph node granulomas of Mycobacterium bovis infected cattle (40). Interestingly, in this species IL-22 was shown to be one of the dominant surrogates of protection from bovine TB after $M$. bovis Bacille-CalmetteGuerin (BCG) vaccination (41). Whether IL-22 is a surrogate of protection from human TB remains to be established.

\section{IL-22 IN HUMAN LATENT AND ACTIVE TB}

In humans Mtb induces a distinct antigen specific IL-22 ${ }^{+} \mathrm{CD} 4^{+}$ $T$ cell population with central memory phenotype, which was first identified in antigen stimulated whole blood from mycobacteria exposed individuals (35). People with latent TB infection (LTBI), who have not progressed to active TB, have significantly higher frequencies of these Mtb specific IL-22 producing $\mathrm{CD} 4^{+}$cells compared to active TB patients (42), which is consistent with the increased frequencies in IFN $\gamma$-producing Th1 cells during LTBI vs. TB. It is likely that both the Th1 and Th22 cell populations in addition to Th17 cells contribute to protection from progression to TB. Furthermore, a single nucleotide polymorphism in the promoter of the IL-22 gene, which is associated with higher Mtb-antigen specific IL-22 production from PBMCs is overrepresented in controls compared to $\mathrm{TB}$ patients suggesting that it is associated with reduced susceptibility to TB (43). Some studies report higher serum concentrations of IL-22 in individuals with LTBI compared to TB patients (44-46), whereas other studies do not show significant differences in circulating IL-22 concentrations (42). These different observations may be due to the ethnic background and Mtb strains prevalent in the respective study cohorts.

At the site of disease however, several studies consistently report increased concentrations of IL-22 in BALF from TB patients compared to controls and higher IL-22 concentrations at the site of disease vs. peripheral blood (35-37), which may be due to migration of antigen specific IL-22 producing $\mathrm{T}$ cells to the site of disease, the lung. In patients with TB pleurisy, IL-22, and IFN $\gamma$ were also elevated in pleural fluid as were antigenspecific IL-22 producing CD4 $+\mathrm{T}$ cells (47). In patients with extra-pulmonary TB-associated pericardial and pleural effusions IL-22 concentrations correlated with MMP-9 expression (36). However, whether IL-22 contributes to immunopathology or is produced to counteract immunopathology was not established in this context, although MMP-9 production has been linked to improved epithelial barrier function in the gut (48) and it is possible that IL-22 and MMP-9 are induced in order to promote healing rather than being drivers of immunopathology. Successful TB treatment restores antigen-specific IL-22 responses by reducing the frequencies of $\mathrm{CD} 19+\mathrm{CD} 1 \mathrm{~d}+\mathrm{CD} 5+$ regulatory B cell, which were shown to suppress IL-22 production (49).

In patients with Mycobacterium avium complex (MAC) infection, low IL-22 concentrations in BALF were associated with a neutrophil dominant inflammatory response, radiological severity and progression to pulmonary MAC disease, whereas individuals high IL-22 concentrations in BALF had greater percentages of lymphocytes and less disease severity (50). This finding is consistent with the observation that IL-22 regulates neutrophilic infiltration as shown in an animal model of lung infection (29). Collectively these data point toward an important role of IL-22 in mycobacterial infection and highlight the need to further define its role in progression from latent to active $\mathrm{TB}$ as well as in treatment outcomes.

\section{IL-22 IN TB-DIABETES CO-MORBIDITY}

The threat of TB and diabetes (T2D) comorbidity to TB control programs is well recognized, but the underlying mechanisms contributing to increased susceptibility of T2D patients to TB and the increased risk of poor treatment outcomes in patients with TB-T2D comorbidity are poorly understood $(51,52)$.

T2D patients with LTBI have lower frequencies of Mtbspecific Th1, Th17, and Th2 responses compared to normoglycemic individuals with LTBI. Once T2D patients develop TB they exhibit higher circulating concentrations of Th1 and Th17 cytokines compared to TB patients without T2D (52). Despite this increased production of Th1 and Th17 cytokines, which are important for protective immune responses to $\mathrm{Mtb}$, TB-T2D patients are more likely to fail treatment and relapse after initial cure (53). Interestingly, IL-22 is the only cytokine 
found at lower concentrations in serum of TB-T2D patients compared to $\mathrm{TB}$ patients without co-morbidities $(44,46)$, but a causative link between low IL-22 serum concentrations and risk of poor treatment outcomes is far from established. Interestingly, Kumar et al. reported that T2D patients with LTBI had higher IL-22 serum concentrations compared to individuals with LTBI and no T2D (45). Although this appears puzzling, it is possible that latently infected T2D patients with high basal concentrations of IL-22 are less likely to progress to active disease.

A study based on a high fat diet mouse model of T2D showed that the induction of IL-22 from $\mathrm{CD}^{+}$cells is impaired in obese mice in response to challenge with the intestinal pathogen Citrobacter rodentium, making them more susceptible to infection. This defect was restricted to IL-22 producing T cells and IL-22 secretion by ILCs was not affected (54). Administration of rIL-22 not only improved mucosal host defense, but also many of the metabolic symptoms including hyperglycemia and insulin resistance in this and another murine T2D model (54, 55). This further raises the question whether IL-22 may be useful as adjunct host-directed therapy in the context of TBT2D.

\section{REFERENCES}

1. Houben RM, Dodd PJ. The global burden of latent tuberculosis infection: a re-estimation using mathematical modelling. PLoS Med. (2016) 13:e1002152. doi: 10.1371/journal.pmed.1002152

2. Barry CE III, Boshoff HI, Dartois V, Dick T, Ehrt S, Young D, et al. The spectrum of latent tuberculosis: rethinking the biology and intervention strategies. Nat Rev Microbiol. (2009) 7:845-55. doi: 10.1038/nrmicro 2236

3. Abel L, Fellay J, Haas DW, Schurr E, Srikrishna G, Urbanowski M, et al. Genetics of human susceptibility to active and latent tuberculosis: present knowledge and future perspectives. Lancet Infect Dis. (2018) 18:e64-75. doi: 10.1016/S1473-3099(17)30623-0

4. Reiling N, Homolka S, Kohl TA, Steinhauser C, Kolbe K, Schutze S, et al. Shaping the niche in macrophages: genetic diversity of the M. tuberculosis complex and its consequences for the infected host. Int J Med Microbiol. (2017) S1438-4221:30294-1. doi: 10.1016/j.ijmm.2017.09.009

5. Ronacher K, Joosten SA, Van Crevel R, Dockrell HM, Walzl G, Ottenhoff TH. Acquired immunodeficiencies and tuberculosis: focus on HIV/AIDS and diabetes mellitus. Immunol Rev. (2015) 264:121-37. doi: 10.1111/imr. 12257

6. Wolk K, Sabat R. Interleukin-22: a novel T- and NK-cell derived cytokine that regulates the biology of tissue cells. Cytokine Growth Factor Rev. (2006) 17:367-80. doi: 10.1016/j.cytogfr.2006.09.001

7. Hansson M, Silverpil E, Linden A, Glader P. Interleukin-22 produced by alveolar macrophages during activation of the innate immune response. Inflamm Res. (2013) 62:561-9. doi: 10.1007/s00011-013-0608-1

8. Eyerich K, Dimartino V, Cavani A. IL-17 and IL-22 in immunity: driving protection and pathology. Eur J Immunol. (2017) 47:607-14. doi: 10.1002/eji.201646723

9. Plank MW, Kaiko GE, Maltby S, Weaver J, Tay HL, Shen W, et al. Th22 cells form a distinct Th lineage from Th17 Cells in vitro with unique transcriptional properties and Tbet-dependent Th1 plasticity. J Immunol. (2017) 198:218290. doi: 10.4049/jimmunol.1601480

10. Wolk K, Witte E, Witte K, Warszawska K, Sabat R. Biology of interleukin22. Semin Immunopathol. (2010) 32:17-31. doi: 10.1007/s00281-0090188-X

\section{CONCLUSIONS}

IL-22 is a key regulator of immunity and inflammation at mucosal surfaces including the lung. Current evidence suggests that an optimal amount of this cytokine prior to infection can contribute to containment of bacteria and to protection from excessive tissue damage. The contribution of IL-22 and Mtbspecific IL-22 ${ }^{+} \mathrm{T}$ cells in protection from progression to TB in presence and absence of $\mathrm{T} 2 \mathrm{D}$ co-morbidity in humans and the importance of this cytokine in $\mathrm{TB}$ treatment response requires further studies.

\section{AUTHOR CONTRIBUTIONS}

$\mathrm{KR}$, RS, and MC reviewed the current literature. KR and RS wrote the manuscript. All authors critically reviewed the manuscript and MC created Figure 1.

\section{FUNDING}

This study was supported by the National Institutes of Health (R01AI116039) and the Mater Foundation to KR.

11. Wolk K, Kunz S, Witte E, Friedrich M, Asadullah K, Sabat R. IL-22 increases the innate immunity of tissues. Immunity (2004) 21:241-54. doi: 10.1016/j.immuni.2004.07.007

12. Ziesche E, Bachmann M, Kleinert H, Pfeilschifter J, Muhl H. The interleukin22/STAT3 pathway potentiates expression of inducible nitric-oxide synthase in human colon carcinoma cells. J Biol Chem. (2007) 282:16006-15. doi: 10.1074/jbc.M611040200

13. Dhiman R, Indramohan M, Barnes PF, Nayak RC, Paidipally P, Rao LV, et al. IL-22 produced by human NK cells inhibits growth of Mycobacterium tuberculosis by enhancing phagolysosomal fusion. J Immunol. (2009) 183:6639-45. doi: 10.4049/jimmunol.0902587

14. Zeng G, Chen CY, Huang D, Yao S, Wang RC, Chen ZW. Membrane-bound IL-22 after de novo production in tuberculosis and anti-Mycobacterium tuberculosis effector function of IL-22+ CD4+ T cells. J Immunol. (2011) 187:190-9. doi: 10.4049/jimmunol.1004129

15. Treerat P, Prince O, Cruz-Lagunas A, Munoz-Torrico M, Salazar-Lezama MA, Selman M, et al. Novel role for IL-22 in protection during chronic Mycobacterium tuberculosis HN878 infection. Mucosal Immunol. (2017) 10:1069-81. doi: 10.1038/mi.2017.15

16. Aujla SJ, Chan YR, Zheng M, Fei M, Askew DJ, Pociask DA, et al. IL-22 mediates mucosal host defense against Gram-negative bacterial pneumonia. Nat Med. (2008) 14:275-81. doi: 10.1038/nm1710

17. Whittington HA, Armstrong L, Uppington KM, Millar AB. Interleukin-22: a potential immunomodulatory molecule in the lung. Am J Respir Cell Mol Biol. (2004) 31:220-6. doi: 10.1165/rcmb.2003-0285OC

18. Hammer AM, Morris NL, Cannon AR, Khan OM, Gagnon RC, Movtchan $\mathrm{NV}$, et al. Interleukin-22 prevents microbial dysbiosis and promotes intestinal barrier regeneration following acute injury. Shock (2017) 48:657-65. doi: 10.1097/SHK.0000000000000900

19. Lin YL, Ip PP, Liao F. CCR6 deficiency impairs IgA production and dysregulates antimicrobial peptide production, altering the intestinal flora. Front Immunol. (2017) 8:805. doi: 10.3389/fimmu.2017. 00805

20. Moyat M, Bouzourene H, Ouyang W, Iovanna J, Renauld JC, Velin D. IL22 -induced antimicrobial peptides are key determinants of mucosal vaccineinduced protection against H. pylori in mice Mucosal Immunol. (2017) 10:27181. doi: $10.1038 / \mathrm{mi} .2016 .38$ 
21. Tyler CJ, Mccarthy NE, Lindsay JO, Stagg AJ, Moser B, Eberl M. Antigenpresenting human gammadelta $\mathrm{T}$ cells promote intestinal CD4(+) $\mathrm{T}$ cell expression of IL-22 and mucosal release of calprotectin. J Immunol. (2017) 198:3417-25. doi: 10.4049/jimmunol.1700003

22. Ivanov S, Renneson J, Fontaine J, Barthelemy A, Paget C, Fernandez EM, et al. Interleukin-22 reduces lung inflammation during influenza A virus infection and protects against secondary bacterial infection. J Virol. (2013) 87:6911-24. doi: 10.1128/JVI.02943-12

23. Dhiman R, Venkatasubramanian S, Paidipally P, Barnes PF, Tvinnereim A, Vankayalapati R. Interleukin 22 inhibits intracellular growth of Mycobacterium tuberculosis by enhancing calgranulin A expression. J Infect Dis. (2014) 209:578-87. doi: 10.1093/infdis/jit495

24. Chellan B, Yan L, Sontag TJ, Reardon CA, Hofmann Bowman MA. IL-22 is induced by S100/calgranulin and impairs cholesterol efflux in macrophages by downregulating ABCG1. J Lipid Res. (2014) 55:443-54. doi: 10.1194/jlr.M044305

25. Pandey AK, Sassetti CM. Mycobacterial persistence requires the utilization of host cholesterol. Proc Natl Acad Sci USA. (2008) 105:4376-80. doi: 10.1073/pnas.0711159105

26. Van Maele L, Carnoy C, Cayet D, Ivanov S, Porte R, Deruy E, et al. Activation of Type 3 innate lymphoid cells and interleukin 22 secretion in the lungs during Streptococcus pneumoniae infection. J Infect Dis. (2014) 210:493-503. doi: $10.1093 /$ infdis/jiu106

27. Trevejo-Nunez G, Elsegeiny W, Conboy P, Chen K, Kolls JK. Critical role of IL-22/IL22-RA1 signaling in pneumococcal pneumonia. J Immunol. (2016) 197:1877-83. doi: 10.4049/jimmunol.1600528

28. Mear JB, Gosset P, Kipnis E, Faure E, Dessein R, Jawhara S, et al. Candida albicans airway exposure primes the lung innate immune response against Pseudomonas aeruginosa infection through innate lymphoid cell recruitment and interleukin-22-associated mucosal response. Infect Immun. (2014) 82:306-15. doi: 10.1128/IAI.01085-13

29. Broquet A, Jacqueline C, Davieau M, Besbes A, Roquilly A, Martin J, et al. Interleukin-22 level is negatively correlated with neutrophil recruitment in the lungs in a Pseudomonas aeruginosa pneumonia model. Sci Rep. (2017) 7:11010. doi: 10.1038/s41598-017-11518-0

30. Guillon A, Jouan Y, Brea D, Gueugnon F, Dalloneau E, Baranek $\mathrm{T}$, et al. Neutrophil proteases alter the interleukin-22-receptordependent lung antimicrobial defence. Eur Respir J. (2015) 46:771-82. doi: 10.1183/09031936.00215114

31. Sharan R, Perez-Cruz M, Kervoaze G, Gosset P, Weynants V, Godfroid F, et al. Interleukin-22 protects against non-typeable Haemophilus influenzae infection: alteration during chronic obstructive pulmonary disease. Mucosal Immunol. (2017) 10:139-49. doi: 10.1038/mi.2016.40

32. Behrends J, Renauld JC, Ehlers S, Holscher C. IL-22 is mainly produced by IFNgamma-secreting cells but is dispensable for host protection against Mycobacterium tuberculosis infection. PLoS ONE (2013) 8:e57379. doi: 10.1371/journal.pone.0057379

33. Wilson MS, Feng CG, Barber DL, Yarovinsky F, Cheever AW, Sher A, et al. Redundant and pathogenic roles for IL-22 in mycobacterial, protozoan, and helminth infections. J Immunol. (2010) 184:4378-90. doi: 10.4049/jimmunol.0903416

34. Qiu L, Huang D, Chen CY, Wang R, Shen L, Shen Y, et al. Severe tuberculosis induces unbalanced up-regulation of gene networks and overexpression of IL-22, MIP-1alpha, CCL27, IP-10, CCR4, CCR5, CXCR3, PD1, PDL2, IL-3, IFN-beta, TIM1, and TLR2 but low antigen-specific cellular responses. J Infect Dis. (2008) 198:1514-9. doi: 10.1086/592448

35. Scriba TJ, Kalsdorf B, Abrahams DA, Isaacs F, Hofmeister J, Black G, et al. Distinct, specific IL-17- and IL-22-producing CD4+ T cell subsets contribute to the human anti-mycobacterial immune response. JImmunol. (2008) 180:1962-70. doi: 10.4049/jimmunol.180.3.1962

36. Matthews K, Wilkinson KA, Kalsdorf B, Roberts T, Diacon A, Walzl $\mathrm{G}$, et al. Predominance of interleukin-22 over interleukin-17 at the site of disease in human tuberculosis. Tuberculosis (2011) 91:587-93. doi: 10.1016/j.tube.2011.06.009

37. Singh S, Maniakis-Grivas G, Singh UK, Asher RM, Mauri F, Elkington PT, et al. Interleukin-17 regulates matrix metalloproteinase activity in human pulmonary tuberculosis. J Pathol. (2018) 244:311-22. doi: 10.1002/path.5013
38. Semple PL, Binder AB, Davids M, Maredza A, Van Zyl-Smit RN, Dheda K. Regulatory $\mathrm{T}$ cells attenuate mycobacterial stasis in alveolar and blood-derived macrophages from patients with tuberculosis. Am J Respir Crit Care Med. (2013) 187:1249-58. doi: 10.1164/rccm.2012101934OC

39. Yao S, Huang D, Chen CY, Halliday L, Zeng G, Wang RC, et al. Differentiation, distribution and gammadelta $\mathrm{T}$ cell-driven regulation of IL-22-producing T cells in tuberculosis. PLoS Pathog. (2010) 6:e1000789. doi: 10.1371/journal.ppat.1000789

40. Palmer MV, Thacker TC, Waters WR. Differential cytokine gene expression in granulomas from lungs and lymph nodes of cattle experimentally infected with aerosolized Mycobacterium bovis. PLOS ONE (2016) 11:e0167471. doi: 10.1371/journal.pone.0167471

41. Bhuju S, Aranday-Cortes E, Villarreal-Ramos B, Xing Z, Singh M, Vordermeier HM. Global gene transcriptome analysis in vaccinated cattle revealed a dominant role of IL-22 for protection against bovine tuberculosis. PLoS Pathog. (2012) 8:e1003077. doi: 10.1371/journal.ppat.10 03077

42. Cowan J, Pandey S, Filion LG, Angel JB, Kumar A, Cameron DW. Comparison of interferon-gamma-, interleukin (IL)-17- and IL-22-expressing CD4 T cells, IL-22-expressing granulocytes and proinflammatory cytokines during latent and active tuberculosis infection. Clin Exp Immunol. (2012) 167:317-29. doi: 10.1111/j.1365-2249.2011.04520.x

43. Zhang G, Chen X, Chan L, Zhang M, Zhu B, Wang L, et al. An SNP selection strategy identified IL-22 associating with susceptibility to tuberculosis in Chinese. Sci Rep. (2011) 1:20. doi: 10.1038/srep0 0020

44. Kumar NP, Sridhar R, Banurekha VV, Jawahar MS, Fay MP, Nutman TB, et al. Type 2 diabetes mellitus coincident with pulmonary tuberculosis is associated with heightened systemic type 1, type 17, and other proinflammatory cytokines. Ann Am Thorac Soc. (2013) 10:441-9. doi: 10.1513/AnnalsATS.201305-112OC

45. Kumar NP, George PJ, Kumaran P, Dolla CK, Nutman TB, Babu S Diminished systemic and antigen-specific Type 1, Type 17, and other proinflammatory cytokines in diabetic and prediabetic individuals with latent Mycobacterium tuberculosis infection. J Infect Dis. (2014) 210:1670-8. doi: 10.1093/infdis/jiu329

46. Kumar NP, Banurekha VV, Nair D, Kumaran P, Dolla CK, Babu S. Type 2 diabetes - Tuberculosis co-morbidity is associated with diminished circulating levels of IL-20 subfamily of cytokines. Tuberculosis (2015) 95:707-12. doi: 10.1016/j.tube.2015.06.004

47. Qiao D, Yang BY, Li L, Ma JJ, Zhang XL, Lao SH, et al. ESAT-6- and CFP-10-specific Th1, Th22 and Th17 cells in tuberculous pleurisy may contribute to the local immune response against Mycobacterium tuberculosis infection. Scand J Immunol. (2011) 73:330-7. doi: 10.1111/j.1365-3083.2011. 02512.x

48. Pujada A, Walter L, Patel A, Bui TA, Zhang Z, Zhang Y, et al. Matrix metalloproteinase MMP9 maintains epithelial barrier function and preserves mucosal lining in colitis associated cancer. Oncotarget (2017) 8:94650-65. doi: 10.18632/oncotarget.21841

49. Zhang M, Zeng G, Yang Q, Zhang J, Zhu X, Chen Q, et al. Antituberculosis treatment enhances the production of IL-22 through reducing the frequencies of regulatory B cell. Tuberculosis (2014) 94:238-44. doi: 10.1016/j.tube.2013.12.003

50. Inomata T, Konno S, Nagai K, Suzuki M, Nishimura M. Neutrophil predominance in bronchoalveolar lavage fluid is associated with disease severity and progression of HRCT findings in pulmonary Mycobacterium avium infection. PLoS ONE (2018) 13:e0190189. doi: 10.1371/journal.pone.0190189

51. Critchley JA, Restrepo BI, Ronacher K, Kapur A, Bremer AA, Schlesinger LS, et al. Defining a research agenda to address the converging epidemics of tuberculosis and diabetes: part 1: epidemiology and clinical management. Chest (2017) 152:165-73. doi: 10.1016/j.chest.2017. 04.155

52. Ronacher K, Van Crevel R, Critchley JA, Bremer AA, Schlesinger LS, Kapur A, et al. Defining a research agenda to address the converging epidemics of tuberculosis and diabetes: part 2: underlying biologic 
mechanisms. Chest (2017) 152:174-80. doi: 10.1016/j.chest.2017. 02.032

53. Baker MA, Harries AD, Jeon CY, Hart JE, Kapur A, Lonnroth K, et al. The impact of diabetes on tuberculosis treatment outcomes: a systematic review. BMC Med. (2011) 9:81. doi: 10.1186/1741-7015-9-81

54. Wang X, Ota N, Manzanillo P, Kates L, Zavala-Solorio J, Eidenschenk C, et al. Interleukin-22 alleviates metabolic disorders and restores mucosal immunity in diabetes. Nature (2014) 514:237-41. doi: 10.1038/nature 13564

55. Hasnain SZ, Borg DJ, Harcourt BE, Tong H, Sheng YH, Ng CP, et al. Glycemic control in diabetes is restored by therapeutic manipulation of cytokines that regulate beta cell stress. Nat Med. (2014) 20:1417-26. doi: 10.1038/nm. 3705
Conflict of Interest Statement: The authors declare that the research was conducted in the absence of any commercial or financial relationships that could be construed as a potential conflict of interest.

The reviewer WB and handling Editor declared their shared affiliation.

Copyright (c) 2018 Ronacher, Sinha and Cestari. This is an open-access article distributed under the terms of the Creative Commons Attribution License (CC BY). The use, distribution or reproduction in other forums is permitted, provided the original author(s) and the copyright owner(s) are credited and that the original publication in this journal is cited, in accordance with accepted academic practice. No use, distribution or reproduction is permitted which does not comply with these terms. 